\title{
ON INTEGRAL TRANSFORM PAIRS ARISING FROM SECOND ORDER DIFFERENTIAL EQUATIONS
}

\author{
by A. MCD. MERCER \\ (Received 5th October 1961)
}

\section{Introduction}

We present in this paper a straight-forward method of deducing integral transform pairs directly from second order differential equations. In (1) this subject has been very thoroughly treated and in view of this we believe that the method described here has its greatest justification in its simplicity.

1. Let $K(\lambda, x)$ and $w(\mu, x)$ be uniquely defined functions of the variables indicated which are, respectively, solutions of the differential equations

and

$$
\frac{1}{p(x)} \frac{d}{d x}\left[p(x) \frac{d K}{d x}\right]+\left[\lambda^{2} u(x)-v(x)\right] K=0
$$

$$
\frac{1}{p(x)} \frac{d}{d x}\left[p(x) \frac{d w}{d x}\right]+\left[-\mu^{2} u(x)-v(x)\right] w=0 .
$$

We shall suppose further that for $\lambda$ and $\mu$ both real and positive $p\left[K^{1} w-w^{1} K\right]$ is a continuous function of $x$ whenever $0 \leqq x<\infty$ and that the expression

$$
\Phi(\lambda, \mu) \equiv\left\{p\left[K^{1} w-w^{1} K\right]\right\}_{x=0}-\left\{p\left[K^{1} w-w^{1} K\right]\right\}_{x=\infty}
$$

exists as a separable function of $\lambda$ and $\mu$. Accordingly we may write

$$
\Phi(\lambda, \mu)=L(\lambda) M(\mu) \text {. }
$$

It would of course be possible, without loss of generality, to take $p=u=1$ in the equations (1.1) and (1.2) and indeed, in view of (1.3) to take $\Phi=1$ but for ease of application it seems preferable to present the analysis in its most general form.

2. Taking the above function $K(\lambda, x)$ we define the $K$-transform of an arbitrary function $f(x)$ by

$$
\tilde{f}(\lambda)=\int_{0}^{\infty} f(x) K(\lambda, x) d x
$$

and we shall assume that there is an inversion formula of the type

$$
f(x)=\int_{0}^{\infty} \tilde{f}(\lambda) H(\lambda, x) d \lambda
$$

It is now our purpose to discover the form of the function $H(\lambda, x)$. 
3. Multiplying (1.1) and (1.2) by $p w$ and $p K$ respectively, subtracting and integrating over the interval $0 \leqq x<\infty$ we have that

$$
\left(\lambda^{2}+\mu^{2}\right) \int_{0}^{\infty} p(x) u(x) w(\mu, x) K(\lambda, x) d x=L(\lambda) M(\mu) .
$$

In view of (2.1) this is to say that we have a one-parameter family of functions

$$
p(x) u(x) w(\mu, x)
$$

whose $K$-transforms are given by

$$
\frac{L(\lambda) M(\mu)}{\lambda^{2}+\mu^{2}}
$$

Hence by the inversion formula (2.2) we have, formally, that

$$
\int_{0}^{\infty} \frac{L(\lambda)}{\lambda^{2}+\mu^{2}} H(\lambda, x) d \lambda=\frac{p(x) u(x) w(\mu, x)}{M(\mu)} .
$$

Now although the pair of integral transform formulae (2.1) and (2.2) may exist it would be possible to choose functions $f(x)$ for which we could find $f(\lambda)$ but such that the inversion formula (2.2) could not be used to recover the original function $f(x)$ due to its diverging. In such a case it may still be possible to recover $f(x)$ by interpreting the integral (2.2) in a wider sense, that is to say by summing it by some regular summation method. It may well be that in some cases the particular functions

$$
p(x) u(x) w(\mu, x)
$$

which we have chosen are of this type and that the integral in (3.2) is divergent in the ordinary sense but that the integral equation (3.2) is still meaningful provided the infinite integral on the left-hand side is summable.

If we introduce the factor

$$
1=\left(\lambda^{2}+\mu^{2}\right) \int_{0}^{\infty} e^{-\left(\lambda^{2}+\mu^{2}\right) p} d p
$$

into the integrand of (3.2) and interchange the orders of integration we have the formal identity

$$
\int_{0}^{\infty} \frac{L(\lambda)}{\lambda^{2}+\mu^{2}} H(\lambda, x) d \lambda=\int_{0}^{\infty} e^{-\mu^{2} p} \int_{0}^{\infty} e^{-\lambda^{2} p} L(\lambda) H(\lambda, x) d \lambda d p .
$$

This identity is valid if the integral on the left is convergent (2) and so in this case we may consider, instead of (3.2), the integral equation

$$
\int_{0}^{\infty} e^{-\mu^{2} p} \int_{0}^{\infty} e^{-\lambda^{2} p} L(\lambda) H(\lambda, x) d \lambda d p=\frac{p(x) u(x) w(\mu, x)}{M(\mu)} .
$$

Now it may happen that the right-hand side of (3.3) exists while the left-hand side does not and in this case (3.3) provides a (clearly regular) method of 
summing the divergent integral

$$
\int_{0}^{\infty} \frac{L(\lambda)}{\lambda^{2}+\mu^{2}} H(\lambda, x) d \lambda
$$

Thus in either case, provided the equation (3.4) is meaningful its solution $H(\lambda, x)$ will be the required kernel of the inversion formula (2.2).

4. It shall be convenient to introduce into (3.4) the substitutions

$$
\begin{gathered}
H_{0}(\lambda, x)=\frac{1}{2} \lambda^{-1} L(\lambda) H(\lambda, x) \\
w_{0}(\mu, x)=\frac{p(x) u(x) w(\mu, x)}{M(\mu)} \\
t=\lambda^{2}, \quad s=\mu^{2}
\end{gathered}
$$

and

the equation now reading

that is

$$
\int_{0}^{\infty} e^{-s p} \int_{0}^{\infty} H_{0}\left(t^{\frac{1}{2}}, x\right) e^{-t p} d t d p=w_{0}\left(s^{\frac{1}{2}}, x\right)
$$

$$
\mathscr{L} \mathscr{L}\left\{H_{0}\left(t^{\frac{1}{2}}, x\right): t \rightarrow p \rightarrow s\right\}=w_{0}\left(s^{\frac{1}{2}}, x\right)
$$

where $\mathscr{L}$ denotes taking the Laplace transform.

It may be mentioned here that in these variables (3.2) reads

$$
\int_{0}^{\infty} \frac{H_{0}\left(t^{\frac{1}{2}}, x\right)}{t+s} d t=w_{0}\left(s^{\frac{1}{2}}, x\right)
$$

so that the replacement of (3.2) by (3.4) is equivalent to replacing the Stieltjes transform of $H_{0}\left(t^{\frac{1}{2}}, x\right)$ by its repeated Laplace transform.

The solution of (4.1) is effected at once by the following lemma.

Lemma. Let $g(s)$ have no singularities other than a branch point at the origin. Let $g(s) \rightarrow 0$ as $|s| \rightarrow \infty$ uniformly with respect to arg $s$ whenever $\frac{\pi}{2} \leqq \arg s \leqq \pi$ and whenever $-\pi \leqq \arg s \leqq-\frac{\pi}{2}$. Furthermore let $\operatorname{sg}(s) \rightarrow 0$ as $|s| \rightarrow 0$ uniformly with respect to arg $s$ whenever $|\arg s|<\pi$.

Then

$$
\mathscr{L} \mathscr{L}\left\{\left[g\left(t e^{i \pi}\right)-g\left(t e^{-i \pi}\right)\right]: t \rightarrow p \rightarrow s\right\}=-2 i \pi g(s) .
$$

The proof of this lemma follows by considering the integral

$$
\int_{C} g(s) e^{p s} d s
$$

where $C$ is the contour shown, and by letting $R \rightarrow \infty, \delta \rightarrow 0$ when we see that

$$
\mathscr{L}^{-1}\{g(s): s \rightarrow p\}=\frac{i}{2 \pi} \mathscr{L}\left\{\left[g\left(t e^{i \pi}\right)-g\left(t e^{-i \pi}\right)\right]: t \rightarrow p\right\}
$$

and hence the result stated.

E.M.S. - E 
On comparing (4.1) and (4.3) it follows that

$$
H_{0}\left(t^{\frac{1}{2}}, x\right)=\frac{i}{2 \pi}\left\{w_{0}\left(t^{\frac{1}{2}} e^{i \pi / 2}, x\right)-w_{0}\left(t^{\frac{1}{2}} e^{-i \pi / 2}, x\right)\right\} .
$$

That is

$$
H(\lambda, x)=\frac{i \lambda}{\pi L(\lambda)} p(x) u(x)\left\{\frac{w\left(\lambda e^{i \pi / 2}, x\right)}{M\left(\lambda e^{i \pi / 2}\right)}-\frac{w\left(\lambda e^{-i \pi / 2}, x\right)}{M\left(\lambda e^{-i \pi / 2}\right)}\right\}
$$

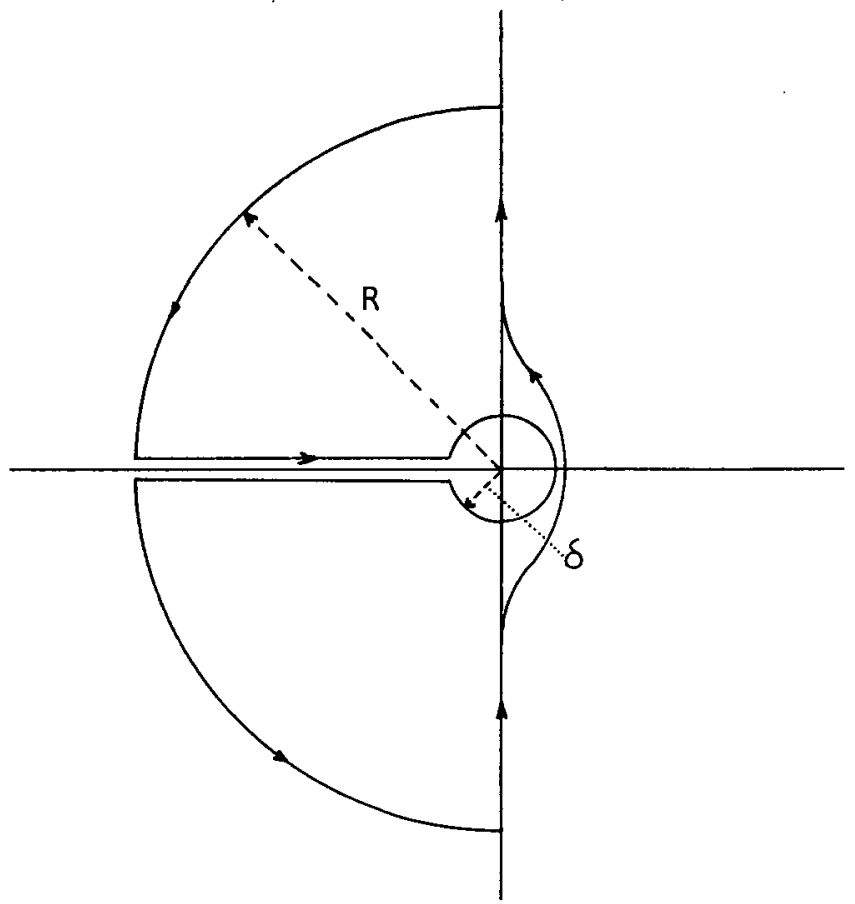

FIG. 1.

5. In this section we consider some particular cases.

Example 1. Take $p=u=1, v=0, K(\lambda, x)=\sin \lambda x$ and $w(\mu, x)=e^{-\mu x}$. We see at once that

$$
L(\lambda)=\lambda, \quad M(\mu)=1 .
$$

Substituting these in (4.4) we find that

$$
H(\lambda, x)=\frac{2}{\pi} \sin \lambda x
$$

This is the Fourier Sine Transform

$$
\tilde{f}(\lambda)=\int_{0}^{\infty} f(x) \sin \lambda x d x
$$




$$
f(x)=\frac{2}{\pi} \int_{0}^{\infty} \tilde{f}(\lambda) \sin \lambda x d \lambda .
$$

\section{Then}

Example 2. Take $p, u, v$ and $w$ as in Example 1 and $K(\lambda, x)=\cos \lambda x$.

$$
L(\lambda)=1, \quad M(\mu)=\mu
$$

and equation (4.4) gives us

$$
H(\lambda, x)=\frac{2}{\pi} \cos \lambda x
$$

being the Fourier Cosine Transform

$$
\begin{aligned}
& \tilde{f}(\lambda)=\int_{0}^{\infty} f(x) \cos \lambda x d x \\
& f(x)=\frac{2}{\pi} \int_{0}^{\infty} \tilde{f}(\lambda) \cos \lambda x d \lambda .
\end{aligned}
$$

In these two examples the integral in (3.2) is convergent and so the question of summability does not arise. In Example 3 this is not the case.

Example 3. Take $p=x, u=1, v=v^{2} / x^{2}, K(\lambda, x)=J_{v}(\lambda x)$ and $w(\mu, x)$ $=K_{v}(\mu x)$. Using the power series and asymptotic developments of the Bessel functions it is soon verified that

$$
L(\lambda)=\lambda^{\nu}, \quad M(\mu)=\mu^{-v}
$$

where to ensure the convergence of the integral in (3.1) it is necessary to take $\operatorname{Re}(v)>-1$.

We have then by (4.4) that

$$
H(\lambda, x)=\frac{i}{\pi} x \lambda^{1-v}\left\{(i \lambda)^{v} K_{v}(i \lambda x)-(-i \lambda)^{v} K_{v}(-i \lambda x)\right\} .
$$

Using the identities (3)

and

$$
K_{v}(z)=\frac{\pi i}{2} e^{i v \pi / 2} H_{v}^{(1)}\left(z e^{i \pi / 2}\right)=-\frac{\pi i}{2} e^{-i v \pi / 2} H_{v}^{(2)}\left(z e^{-i \pi / 2}\right)
$$

this easily reduces to

$$
J_{v}(z)=\frac{1}{2}\left[H_{v}^{(1)}(z)+H_{v}^{(2)}(z)\right]
$$

$$
H(\lambda, x)=x \lambda J_{v}(\lambda x)
$$

giving the Hankel Transform of order $v$

$$
\begin{aligned}
& \tilde{f}(\lambda)=\int_{0}^{\infty} f(x) J_{v}(\lambda x) d x \\
& f(x)=x \int_{0}^{\infty} \tilde{f}(\lambda) \lambda J_{v}(\lambda x) d \lambda .
\end{aligned}
$$

It may now be verified that the integral in (3.2) is convergent if $\operatorname{Re}(v)<\frac{3}{2}$ and no question of summability arises. However if the meaning of (3.2) is so 
extended then we need merely have that $\operatorname{Re}(v)<\infty$. In both cases of course it is necessary for the reason mentioned above to have $\operatorname{Re}(v)>-1$.

Example 4. Take $p=x, u=1 / x^{2}, v=1, K(\lambda, x)=K_{i \lambda}(x)$ and $w(\mu, x)$ $=I_{\mu}(x)$. Again it is not difficult to see that

$$
L(\lambda)=M(\mu)=1
$$

and substitution into (4.4) yields

$$
\begin{aligned}
H(\lambda, x) & =\frac{i \lambda}{\pi x}\left\{I_{i \lambda}(x)-I_{-i \lambda}(x)\right\} \\
& =\frac{2 \lambda}{\pi^{2} x} \sinh \lambda \pi K_{i \lambda}(x) .
\end{aligned}
$$

This is the Kontorovich-Lebedev transform (4)

$$
\begin{aligned}
& f(\lambda)=\int_{0}^{\infty} f(x) K_{i \lambda}(x) d x \\
& f(x)=\frac{2}{\pi^{2}} x^{-1} \int_{0}^{\infty} f(\lambda) \lambda \sinh \lambda \pi K_{i \lambda}(x) d \lambda .
\end{aligned}
$$

In this case the integral in (3.2) is divergent but is summable by the method described so that the equation (3.4) is meaningful.

In each of these examples it is not difficult to verify that the other hypotheses, in particular those of the lemma, are satisfied.

\section{Conclusion}

It would be possible to enlarge considerably on the number of examples given but little would be gained in doing so. One point which must be mentioned is that all our analysis proceeds from the assumption that there is an inversion formula of the type (2.2). No attempt has been made to prove the existence of such a formula and in each particular case this would require a separate investigation.

\section{REFERENCES}

(1) E. C. Titchmarsh, Eigenfunction Expansions (Oxford University Press, London, 1946).

(2) T. J. I'A. ВRомwich, Theory of Infinite Series (1926).

(3) Higher Transcendental Functions, The Bateman Manuscript Project, Vol. 2, McGraw-Hill (1953).

(4) M. J. Kontorovich and N. N. LeBedev, J. Exper. Theor. Phys. U.S.S.R., 8 (1938), 1192-1206.

THE QUEEN'S UNIVERSITY

BELFAST 